\title{
ON CONFORMAL TRANSFORMATION OF CERTAIN FINSLER SPACES
}

\section{IIIII}

\author{
S. C. RASTOGI AND A. K. DWIVEDI
}

\section{Introduction}

M. S. Knebelman [4] first defined the conformal theory of Finsler metrics, such that two metric functions $L$ and $\underline{L}$ are conformal if the length of an arbitrary vector in the space with the metric $L$ is proportional to the length in the space with metric $\underline{L}$, that is, if $\underline{g}_{i j}=\phi g_{i j}$, where $g_{i j}$ and $\underline{g}_{i j}$ are the metric tensors corresponding to metric functions $L$ and $\underline{L}$ respectively and $\phi$ is a function of coordinates. Conformal transformations in Finsler spaces have further been studied by various authors namely Hashiguchi [1], Izumi [2, 3], Matsumoto [8] and others. The purpose of the present paper is to sutdy conformal transformation of $L(\alpha, \beta)$-metric (Matsumoto [5, 7] ) and its special case related to Randers' space [9]. Throughout the present paper we shall follow the notations used in Matsumoto's monograph [6].

\section{Preliminaries}

Let $\left(M^{n}, L\right)$ be an $n$-dimensional Finsler space equipped with the fundamental function $L(x, y)$ on a differentiable manifold $M^{n}$. Let $\left(M^{n},{ }^{*} L\right)$ be another Finsler space equipped with the fundamental function ${ }^{*} L(x, y)$ such that Matsumoto [5]:

$$
{ }^{*} L(x, y)=L(x, y)+\beta(x, y) .
$$

where $\beta(x, y)=b_{i}(x) d x^{i}$.

We also have for $l_{i}=\partial L / \partial y^{i},{ }^{*} l_{i}=\partial^{*} L / \partial y^{i}$ and $b_{i}=\partial \beta / \partial y^{i}$,

$$
{ }^{*} l_{i}=l_{i}+b_{i} .
$$

If $h_{i j}=g_{i j}-l_{i} l_{j}=L L_{i j}$ we have ${ }^{*} h_{i j} /{ }^{*} L=h_{i j} / L$ or $L_{i j}={ }^{*} L_{i j}$ such that [5]

$$
{ }^{*} g_{i j}=\tau\left(g_{i j}-l_{i} l_{j}\right)+{ }^{*} l_{i}{ }^{*} l_{j},{ }^{*} g^{i j}=\tau^{-1} g^{i j}+\mu l^{i} l^{j}-\tau^{-2}\left(l^{i} b^{j}+l^{j} b^{i}\right),
$$

where $\mu=\left(L b^{2}+\beta\right) /\left({ }^{*} L \tau^{2}\right), b^{2}=b_{i} b^{i}, b^{i}=g^{i j} b_{j}, \tau={ }^{*} L / L$.

Received October 21, 2004.

2000 Mathematics Subject Classification. 53B40.

Key words and phrases. Conformal transformation, Finsler space, Randers' space. 
Since ${ }^{*} l^{i}=\tau^{-1} l^{i}$, therefore for $m_{i}=b_{i}-(\beta / L) l_{i}$, we can obtain

$$
{ }^{*} C_{i j k}=\tau C_{i j k}+\left(h_{i j} m_{k}+h_{j k} m_{i}+h_{k i} m_{j}\right) / 2 L .
$$

The $h$ - and $v$-covariant derivatives of a covariant vector field $X_{i}$ are defined as

$$
X_{i \mid j}=\partial_{j} X_{i}-N_{j}^{r}\left(\Delta_{r} X_{i}\right)-X_{r} F_{i j}^{r}
$$

and

$$
X_{i \mid j}=\Delta_{j} X_{i}-X_{r} C_{i j}^{r},
$$

where the symbol $\partial_{j}$ and $\Delta_{j}$ stands for $\partial / \partial x^{j}$ and $\partial / \partial y^{j}$ respectively, $\left(F_{j k}^{i}, N_{j}^{i}, C_{j k}^{i}\right)$ are connection parameters of $F^{n}$ such that $N_{k}^{i}=F_{0 k}^{i}=y^{r} F_{r k}^{i}$ and $C_{j k}^{r}=g^{i r} C_{i j k}$.

If ${ }^{*} F_{j k}^{i}$ denotes the Cartan's connection of ${ }^{*} F^{n}$, then it is given by Matsumoto [5]

$$
{ }^{*} F_{j k}^{i}=F_{j k}^{i}+D_{j k}^{i},
$$

where $D_{j k}^{i}$ is a tensor of type $(1,2)$ such that it satisfies [5]

$$
\begin{gathered}
L_{i j r} D_{o k}^{r}+L_{r j} D_{i k}^{r}+L_{i r} D_{j k}^{r}=0, L_{r i} D_{o j}^{r}+\left(l_{r}+b_{r}\right) D_{i j}^{r}=b_{i \mid j}, \\
D_{00}^{i}=2 L F_{0}^{i}+\tau^{-1}\left(E_{00}-2 L F_{r 0} b^{r}\right) l^{i} \\
D_{0 j}^{i}=L G_{j}^{i}+\tau^{-1} l^{i}\left(G_{j}-L G_{m j} b^{m}\right)
\end{gathered}
$$

and

$$
D_{j k}^{i}=L H_{r j k}\left(g^{i r}-l^{i} b^{r} \tau^{-1}\right)+l^{i} \tau^{-1} H_{j k},
$$

where

$$
\begin{gathered}
2 F_{j k}=b_{j \mid k}-b_{k \mid j}, \quad 2 E_{j k}=b_{j \mid k}+b_{k \mid j} . \\
G_{i j}=F_{i j}-L_{i j r} D_{00}^{r} / 2, \quad G_{j}=E_{j 0}-L_{j r} D_{00}^{r} / 2, \quad G_{j}^{i}=g^{i r} G_{r j} \\
H_{i j k}=\left(L_{j k r} D_{0 i}^{r}-L_{k i r} D_{0 j}^{r}-L_{i j r} D_{0 k}^{r}\right) / 2
\end{gathered}
$$

and

$$
H_{j k}=E_{j k}-\left(L_{j r} D_{0 k}^{r}+L_{k r} D_{0 j}^{r}\right) / 2 .
$$

The $T$-tensor in a Finsler space is defined by [6]:

$$
T_{h i j k}=L C_{h i j \mid k}+C_{h i j} l_{k}+C_{h i k} l_{j}+C_{h j k} l_{i}+C_{i j k} l_{h},
$$

which in a space with generalized $(\alpha, \beta)$ metric can be expressed as $[5]$

$$
{ }^{*} T_{h i j k}={ }^{*} L^{*} C_{h i j \mid k}+{ }^{*} C_{h i j}{ }^{*} l_{k}+{ }^{*} C_{h i k}{ }^{*} l_{j}+{ }^{*} C_{h j k}{ }^{*} l_{i}+{ }^{*} C_{i j k}{ }^{*} l_{h} .
$$




\section{Conformal transformation}

Let us assume that there exists a conformal transformation of Finsler spaces which transform in such away that $\underline{L}=L e^{\sigma},(\sigma=\sigma(x)),{ }^{*} \underline{L}={ }^{*} L e^{\sigma}$. From equation (1.1), (1.2), (1.3) and (1.4) we can obtain for $\underline{\tau}=\tau, \underline{\mu}=\mu$,

$$
\begin{gathered}
\underline{l}_{i}=l_{i} e^{\sigma}, \underline{l}^{i}=l^{i} e^{-\sigma},{ }^{*} \underline{l}_{i}={ }^{*} l_{i} e^{\sigma},{ }^{*} \underline{l}^{i}={ }^{*} l^{i} e^{-\sigma}, \underline{b}_{i}=b_{i} e^{\sigma}, \underline{b}^{i}=b^{i} e^{-\sigma}, \\
\underline{\beta}=\beta e^{\sigma}, \underline{y}_{i}=y_{i} e^{2 \sigma}, \underline{y}^{i}=y^{i},{ }^{*} \underline{y}_{i}=e^{2 \sigma *} y_{i},{ }^{*} \underline{y}^{i}={ }^{*} y^{i}, \\
\underline{g}_{i j}=g_{i j} e^{2 \sigma},{ }^{*} \underline{g}_{i j}={ }^{*} g_{i j} e^{2 \sigma},{ }^{*} \underline{h}_{i j}=\tau e^{2 \sigma} h_{i j},{ }^{*} \underline{L}_{i j}=L_{i j} e^{\sigma}, \\
\underline{g}^{i j}=g^{i j} e^{-2 \sigma},{ }^{*} \underline{g}^{i j}={ }^{*} g^{i j} e^{-2 \sigma},{ }^{*} \underline{h}^{i j}={ }^{*} h^{i j} e^{-2 \sigma},
\end{gathered}
$$

and

$$
\underline{C}_{i j k}=C_{i j k} e^{2 \sigma},{ }^{*} \underline{C}_{i j k}={ }^{*} C_{i j k} e^{2 \sigma},{ }^{*} \underline{L}_{i j k}=L_{i j k} e^{\sigma} \text { and }\left(\underline{\Delta}_{k} \underline{h}_{i j}\right)=e^{2 \sigma}\left(\Delta_{k} h_{i j}\right) .
$$

From equations (2.1), (2.2), (2.3) and (2.4) we can obtain

Theorem 2.1. Under the given conformal transformation following entities are conformally invariant ${ }^{*} l_{i}{ }^{*} L^{-1} ;{ }^{*} L^{*} l^{i} ; L^{-1} b_{i} ; L b^{i} ; L^{-1} \beta ;{ }^{*} g_{i j} L^{-2} ;{ }^{*} g^{i j *} L^{2} ;{ }^{*} h_{i j}{ }^{*} L^{-2}$; ${ }^{*} h^{i j *} L^{2} ;{ }^{*} C_{i j k}{ }^{*} L^{-2}$.

We know that Izumi [2, 3]

$$
\underline{G}^{i}=G^{i}+B^{i h} \sigma_{h}, \quad \underline{G}_{j}^{i}=G_{j}^{i}+b_{j}^{i}, \quad \underline{G}_{j k}^{i}=G_{j k}^{i}+b_{j k}^{i},
$$

where

$$
B^{i h}=y^{i} y^{h}-L^{2} g^{i h} / 2, \quad b_{j}^{i}=\left(\Delta_{j} B^{i h}\right) \sigma_{h}, \quad b_{j k}^{i}=\left(\Delta_{k}\left(\Delta_{j} B^{i h}\right)\right) \sigma_{h} .
$$

From equation (1.12) and $F_{0}^{i}=F_{j 0} g^{i j}$ we can obtain

and

$$
\begin{aligned}
\underline{N}_{j}^{i} & =N_{j}^{i}+b_{j}^{i}, \\
2 \underline{F}_{j k} & =e^{\sigma}\left[2 F_{j k}+b_{j} \sigma_{k}-b_{k} \sigma_{j}\right], \\
2 \underline{E}_{j k} & =e^{\sigma}\left[2 E_{j k}+b_{j} \sigma_{k}+b_{k} \sigma_{j}\right]
\end{aligned}
$$

$$
\underline{F}_{0}^{i}=e^{-\sigma}\left[F_{0}^{i}+\left(b^{i} \sigma_{0}-\beta g^{i j} \sigma_{j}\right) / 2\right] .
$$

From $\underline{L}=L e^{\sigma}$, we can write $\log \underline{L}=\log L+\sigma$, which gives

$$
\sigma_{k}=\underline{L}^{-1}\left(\partial_{k} \underline{L}\right)-L^{-1}\left(\partial_{k} L\right), \quad \sigma_{0}=\left\{\underline{L}^{-1}\left(\partial_{k} \underline{L}\right)-L^{-1}\left(\partial_{k} L\right)\right\} y^{k} .
$$

Hence from equations (2.8), (2.9) and (2.10) we have:

Theorem 2.2. Under the given conformal transformation following entities are conformally invariant: 

(a) $\left(b_{j} \sigma_{k}-b_{k} \sigma_{j}\right) / L$;
(b) $\left(b_{j} \sigma_{k}+b_{k} \sigma_{j}\right) / L$;
(c) $L\left(b^{i} \sigma_{0}-\beta g^{i j} \sigma_{j}\right)$;
(d) $L^{-1}\left[2 F_{j k}-L^{-1}\left(b_{j} \partial_{k} L-b_{k} \partial_{j} L\right)\right]$;
(e) $L^{-1}\left[2 E_{j k}-L^{-1}\left(b_{j} \partial_{k} L+b_{k} \partial_{j} L\right)\right]$;
(f) $L\left[F_{0}^{i}-(1 / 2)\left(\partial_{j} L\right)\left(b^{i} y^{i}-\beta g^{i j}\right)\right]$.

From equation (1.9) we can obtain

$$
\underline{D}_{00}^{i}=D_{00}^{i}+B_{00}^{i}
$$

where

$$
B_{00}^{i}=:\left\{L\left(\sigma_{0} b^{i}-\beta \sigma_{p} g^{p i}\right)-y^{i} \tau^{-1}\left(b^{2} \sigma_{0}-\beta \sigma_{p} b^{p}-L^{-1} \beta \sigma_{0}\right)\right\} / 2 .
$$

Equation (2.12) with the help of (2.13) gives on simplification

Theorem 2.3. Under the given conformal transformation tensor $D_{00}^{* r}$ defined by

$$
D_{00}^{* r}=: D_{00}^{r}-\left(\partial_{k} L\right)\left[b^{r} y^{k}-\beta g^{k r}-l^{r} \tau^{-1}\left\{\left(b^{2}-\beta L^{-1}\right) y^{k}-\beta b^{k}\right\}\right] / 2
$$

is conformally invariant.

From equation (1.13) we get

$$
\underline{G}_{i j}=e^{\sigma}\left[G_{i j}+\left(b_{i} \sigma_{j}-b_{j} \sigma_{i}\right) / 2-L_{i j r} B_{00}^{r}\right],
$$

and

$$
\underline{G}_{j}=e^{\sigma}\left[G_{j}+\left(b_{j} \sigma_{0}+\beta \sigma_{j}\right) / 2-L_{j r} B_{00}^{r}\right] .
$$

Since $G_{j}=E_{j 0}-F_{j 0}$, we can also obtain

$$
\underline{G}_{j}=e^{\sigma}\left(G_{j}+\beta \sigma_{j}\right) .
$$

Comparing equations (2.16a) and (2.16b), we get

$$
L_{j r} B_{00}^{r}=\left(b_{j} \sigma_{0}-\beta \sigma_{j}\right) / 2 .
$$

From equations (2.15) and (2.17), we get

$$
\underline{G}_{i j}=e^{\sigma}\left[G_{i j}-L_{i r}\left(\Delta_{j} B_{00}^{r}\right)\right] .
$$

From equations (2.15) and (2.16), we can obtain

Theorem 2.4. Under the given conformal transformation following entities are conformally invariant:

(a) $L^{-1}\left\{G_{k}-\beta L^{-1}\left(\partial_{k} L\right)\right\}$,

(b) $L^{-1}\left[G_{i j}-L^{-1}\left\{b_{i}\left(\partial_{j} L\right)-b_{j}\left(\partial_{i} L\right)\right\}\right]-L^{-1} L_{i j r}\left(\partial_{k} L\right)\left[b^{r} y^{k}-\beta g^{k r}-l^{r} \tau^{-1}\left\{\left(b^{2}-\beta L^{-1}\right) y^{k}-\right.\right.$ $\left.\left.\beta b^{k}\right\}\right] / 2$. 
With the help of equations (1.10), (2.17) and (2.18) we can obtain

$$
\underline{D}_{0 j}^{i}=D_{0 j}^{i}+B_{0 j}^{i} .
$$

where

$$
B_{0 j}^{i}=: \tau^{-1} l^{i} \beta \sigma_{j}-\left(\Delta_{j} B_{00}^{r}\right)\left(h_{r}^{i}-\tau^{-1} l^{i} m_{r}\right) .
$$

From equations (2.19) and (2.20) with the help of (2.12), we can obtain

Theorem 2.5. Under the given conformal transformation tensor $D_{0 j}^{* i}$ defined by

$$
D_{0 j}^{* i}=: D_{0 j}^{i}-\left[\tau^{-1} l^{i} \beta L^{-1}\left(\partial_{j} L\right)-\left(\Delta_{j} D_{00}^{r}\right)\left(h_{r}^{i}-\tau^{-1} l^{i} m_{r}\right)\right],
$$

is conformally invariant.

Multiplying (2.19) by $y^{j}$ comparing the resulting equation with (2.12) and using (2.13) we obtain on simplification

$$
L\left[\beta \sigma_{p}\left(g^{p i}-l^{i} b^{p} \tau^{-1}\right)-\sigma_{0}\left(b^{i}-l^{i} b^{2} \tau^{-1}\right)\right]=\left(\Delta_{j} P^{r}\right)\left(h_{r}^{i}-\tau^{-1} l^{i} m_{r}\right) y^{j},
$$

which implies

Theorem 2.6. Under the given conformal transformation, there exists a scalar $\sigma(x)$, which satisfies equation (2.22).

Since from equation (1.13) we can obtain

$$
G_{k j}=\Delta_{k} G_{j}-\left(l_{r}+b_{r}\right)\left(\Delta_{k} D_{0 j}^{r}\right),
$$

therefore by virtue of equations (2.14) and (2.23) we can obtain on simplification

$$
\left(l_{r}+b_{r}\right) \Delta_{k} B_{0 j}^{r}-L_{j k r} B_{00}^{r}=E_{j k}
$$

and

$$
\Delta_{k}\left\{\left(\Delta_{j} B_{00}^{t}\right) A_{t}^{r}\right\}=\left(l_{r}+b_{r}\right) \Delta_{k}\left(\tau^{-1} l^{r} \beta \sigma_{j}\right)-L_{k r}\left(\Delta_{j} B_{00}^{r}\right)-b_{k} \sigma_{j},
$$

where $A_{r}^{i}=\left(h_{r}^{i}-\tau^{-1} l^{i} L^{-1} m_{r}\right)$.

Hence we have:

Theorem 2.7. Under the given conformal transformation, there exists a scalar $\sigma(x)$, for which the tensors $B_{00}^{r}$ and $A_{r}^{i}$ satisfy (2.24).

From equations (1.14) and (1.15), we can obtain

$$
\underline{H}_{i j k}=e^{\sigma}\left[H_{i j k}+(1 / 2)\left\{L_{j k r} B_{0 i}^{r}-L_{k i r} B_{0 j}^{r}-L_{i j r} B_{0 k}^{r}\right\}\right],
$$

and

$$
\underline{H}_{j k}=e^{\sigma}\left[H_{j k}+\left\{b_{j} \sigma_{k}+b_{k} \sigma_{j}+L_{j r}\left(\Delta_{k} B_{00}^{r}\right)+L_{k r}\left(\Delta_{j} B_{00}^{r}\right)\right\}\right] .
$$


From equations (2.25) and (2.26) on simplification we can obtain

Theorem 2.8. Under the given conformal transformation, following entities are conformally invariant

$$
\begin{aligned}
& L^{-1}\left[\tau^{-1} \beta\left(\partial_{i} L\right) L^{-1}+\left(\Delta_{i} D_{00}^{r}\right)\left(l_{r}+\tau^{-1} m_{r}\right)\right], \\
& L^{-1}\left[H_{j k}-\left(L_{j r} \Delta_{k} D_{00}^{r}+L_{k r} \Delta_{j} D_{00}^{r}\right)-L^{-1}\left(b_{k} \partial_{j} L+b_{j} \partial_{k} L\right)\right], \\
& L^{-1}\left[H_{i j k}-(1 / 2)\left\{L_{j k r} D_{0 i}^{r}-L_{k i r} D_{0 j}^{r}-L_{i j r} D_{0 k}^{r}\right\}\right] .
\end{aligned}
$$

From equation (1.11), we can obtain

$$
\underline{D}_{j k}^{i}=D_{j k}^{i}+B_{j k}^{i}
$$

where

$$
\begin{array}{r}
B^{i}{ }_{j k}=:( \\
(1 / 2) L\left\{L_{j k t} B_{0 r}^{t}-L_{k r t} B_{0 j}^{t}-L_{j r t} B_{0 k}^{t}\right\}\left(g^{i r}-l^{i} b^{r} \tau^{-1}\right) \\
+l^{i} \tau^{-1}\left\{b_{j} \sigma_{k}+b_{k} \sigma_{j}+L_{j r}\left(\Delta_{k} B_{00}^{r}\right)+L_{k r}\left(\Delta_{j} B_{00}^{r}\right)\right\} .
\end{array}
$$

With the help of equations (2.28) and (2.29), we can obtain

Theorem 2.9. Under the given conformal transformation, the tensor defined by

$$
\begin{array}{r}
D_{j k}^{* i}=: D_{j k}^{i}-(1 / 2) L\left\{L_{j k t} D_{0 r}^{t}-L_{k r t} D_{0 j}^{t}-L_{r j t} D_{0 k}^{t}\right\}\left(g^{i r}-l^{i} b^{r} \tau^{-1}\right) \\
\left.-l^{i} \tau^{-1}\left\{L^{-1}\left(b_{j} \partial_{k} L+b_{k} \partial_{j} L\right)+L_{j r} \Delta_{k} D_{00}^{r}\right)+L_{k r} \Delta_{j} D_{00}^{r}\right\}
\end{array}
$$

is conformally invariant.

From equations (1.16) and (1.17), we can easily obtain ${ }^{*} \underline{T}_{h i j k}=e^{3 \sigma *} T_{h i j k}$, which implies

Theorem 2.10. Under the given conformal transformation, the tensor ${ }^{*} L^{-3 *} T_{\text {hijk }}$ is conformally invariant.

\section{Conformal transformation of connection parameters}

From equation (1.7) with the help of equation (2.28), (2.29) and Hashiguchi [1]

$$
\underline{F}_{j k}^{i}=F_{j k}^{i}+U_{j k}^{i}
$$

where

$$
U_{j k}^{i}=\delta_{j}^{i} \sigma_{k}+\delta_{k}^{i} \sigma_{j}+C_{j m}^{i} B_{k}^{m}+C_{k m}^{i} B_{j}^{m}-g^{i n} C_{j k m} B_{n}^{m}-g_{j k} \sigma^{i},
$$

we can obtain

$$
{ }^{*} \underline{F}_{j k}^{i}={ }^{*} F_{j k}^{i}+{ }^{*} U_{j k}^{i}
$$


where

$$
\begin{array}{r}
{ }^{*} U_{j k}^{i}=:\left[\delta_{j}^{i} \sigma_{k}+\delta_{k}^{i} \sigma_{j}+C_{j m}^{i} B_{k}^{m}+C_{k m}^{i} B_{j}^{m}-g^{i n} C_{j k m} B_{n}^{m}-g_{j k} \sigma^{i}\right. \\
+(1 / 2) L\left(L_{j k t} B_{0 r}^{t}-L_{k r t} B_{0 j}^{t}-L_{j r t} B_{0 k}^{t}\right)\left(g^{i r}-l^{i} b^{r} \tau^{-1}\right) \\
\left.+l^{i} \tau^{-1}\left\{b_{j} \sigma_{k}+b_{k} \sigma_{j}+L_{j r}\left(\Delta_{k} B_{00}^{r}\right)+L_{k r}\left(\Delta_{j} B_{00}^{r}\right)\right\}\right] .
\end{array}
$$

From equation (3.3) on multiplication by $y^{j}$ we can obtain by virtue of ${ }^{*} U^{i}{ }_{j k} y^{j}=:{ }^{*} b^{i}{ }_{k}$

$$
{ }^{*} \underline{N}_{k}^{i}={ }^{*} N_{k}^{i}+{ }^{*} b_{k}^{i},
$$

where

$$
\begin{aligned}
{ }^{*} b_{k}^{i}=: & {\left[y^{i} \sigma_{k}+\delta_{k}^{i} \sigma_{0}+L^{2} \sigma^{m} C_{k m}^{i}-y_{k} \sigma^{i}-(1 / 2) L\left(L_{k t} B_{0 r}^{t}+L_{k r t} B_{00}^{t}\right.\right.} \\
& \left.\left.-L_{r t} B_{0 k}^{t}\right)\left(g^{i r}-l^{i} b^{r} \tau^{-1}\right)+l^{i} \tau^{-1}\left\{\beta \sigma_{k}+b_{k} \sigma_{0}+L_{k r}\left(\Delta_{j} B_{00}^{r}\right) y^{j}\right\}\right] .
\end{aligned}
$$

Hence we have:

Theorem 3.1. Under the given conformal transformation in a space with generalized $(\alpha, \beta)$-metric the entities ${ }^{*} U_{j k}^{i}$ and ${ }^{*} b_{k}^{i}$ given by $(3.4)$ and (3.6) respectively are conformally invariant.

From equations (3.3), (3.4), (3.5) and (3.6) we can obtain

$$
N_{k}^{* i}=:{ }^{*} N_{k}^{i}+{ }^{*} M_{k}^{i}
$$

and

$$
F^{* i}{ }_{j k}=:{ }^{*} F_{j k}^{i}+{ }^{*} M_{j k}^{i},
$$

where

$$
\begin{aligned}
{ }^{*} M_{k}^{i}=: & (1 / 2) L\left(L_{k t} D_{0 r}^{t}+L_{k r t} D_{00}^{t}-L_{r t} D_{0 k}^{t}\right)\left(g^{i r}-l^{i} b^{r} \tau^{-1}\right) \\
& -\left(\partial_{k} L\right) L^{-1}\left(y^{i}+L^{2} C_{r m}^{i} g^{r m}+l^{i} \tau^{-1} \beta\right) \\
& -\left(\partial_{r} L\right)\left(L^{-1} y^{r} \delta_{k}^{i}-y_{k} g^{i r}+l^{i} l^{r} \tau^{-1} b_{k}\right)-l^{i} \tau^{-1} L_{k r}\left(\Delta_{j} D_{00}^{r}\right) y^{j}
\end{aligned}
$$

and

$$
\begin{aligned}
{ }^{*} M_{j k}^{i}=: & -L^{-1}\left(\delta_{k}^{i} \partial_{j} L+\delta_{j}^{i} \partial_{k} L\right)-L^{-1}\left(\partial_{r} L\right)\left\{C_{k m}^{i}\left(y_{j} g^{r m}-\delta_{j}^{m} y^{r}-L^{2} C_{j}^{m r}\right)\right. \\
& +C_{j m}^{i}\left(y_{k} g^{r m}-\delta_{k}^{m} y^{r}-L^{2} C_{k}^{m r}\right)+g^{i n} C_{j k m}\left(y_{n} g^{r m}-\delta_{n}^{m} y^{r}-L^{2} C_{n}^{m r}\right) \\
& \left.+g_{j k} g^{r i}\right\}-(1 / 2) L\left\{\left(L_{j k t} D_{0 r}^{t}-L_{k r t} D_{0 j}^{t}-L_{j r t} D_{0 k}^{t}\right)\left(g^{i r}-l^{i} b^{r} \tau^{-1}\right)\right\} \\
& -l^{i} \tau^{-1}\left\{L\left(b_{j} \partial_{k} L+b_{k} \partial_{j} L\right)+L_{j r}\left(\Delta_{k} D_{00}^{r}\right)+L_{k r}\left(\Delta_{j} D_{00}^{r}\right)\right\} .
\end{aligned}
$$

Theorem 3.2. Under the given conformal transformation in a space with generalized $(\alpha, \beta)$-metric the entities defined by $N_{k}^{* i}$ and $F_{j k}^{* i}$ are conformally invariant. 


\section{Conformal transformation of torsion and curvature tensors}

The $h$-torsion tensor $R_{j k}^{i}$ is expressed as [6]:

$$
R_{j k}^{i}=\mathrm{C}_{(j, k)}\left\{\partial_{k} N^{i}{ }_{j}-N_{k}^{r} \Delta_{r} N_{j}^{i}\right\},
$$

therefore by virtue of ${ }^{*} N_{j}^{i}=N_{j}^{i}+D_{0 j}^{i}$, we can easily obtain

$$
{ }^{*} R_{j k}^{i}=R_{j k}^{i}+I_{j k}^{i},
$$

where

$$
I^{i}{ }_{j k}=: \mathrm{C}_{(j, k)}\left[D_{0 j \mid k}^{i}+D_{0 j}^{m}\left\{\left(\Delta_{m}{ }^{*} F_{s k}^{i}\right) y^{s}+D_{m k}^{i}\right\}\right]
$$

and $\mathrm{C}_{(j, k)}$ means interchange of $j$ and $k$ and subtraction.

From equation (4.2) it is easy to get

$$
{ }^{*} \underline{R}_{j k}^{i}={ }^{*} R_{j k}^{i}+J_{j k}^{i},
$$

where

$$
J_{j k}^{i}=: \complement_{(j, k)}\left[{ }^{*} b_{j \| k}^{i}+{ }^{*} b_{j}^{m}\left(\Delta_{m}{ }^{*} N_{k}^{i}-{ }^{*} F_{m k}^{i}\right)\right]
$$

and symbol $\| k$, means covariant derivative corresponding to ${ }^{*} F_{j k}^{i}$.

The $h v$-torsion tensor $P_{j k}^{i}$ is expressed as [6]:

$$
P_{j k}^{i}=\Delta_{k} N_{j}^{i}-F_{j k}^{i},
$$

therefore we can obtain

$$
{ }^{*} P_{j k}^{i}=P_{j k}^{i}+\Delta_{k} D_{0 j}^{i}-D_{j k}^{i},
$$

which on conformal transformation gives

$$
{ }^{*} \underline{P}_{j k}^{i}={ }^{*} P_{j k}^{i}+\Delta_{k}{ }^{*} b_{j}^{i}+{ }^{*} U_{j k}^{i} .
$$

Hence we have:

Theorem 4.1. The torsion tensors of a space with generalized $(\alpha, \beta)$-metric, when conformally transformed, satisfy equations (4.4) and (4.8) such that entities $J_{j k}^{i}$ and $\left(\Delta_{k}{ }^{*} b_{j}^{i}+{ }^{*} U_{j k}^{i}\right)$ are conformally invariant.

Further with the help of equations (3.7), (3.8), (3.9), (3.10), (4.4) and (4.5), we can define

$$
R^{* i}{ }_{j k}=:{ }^{*} R_{j k}^{i}+\mathrm{C}_{(j, k)}\left({ }^{*} M_{j \| k}^{i}+2^{*} M_{j}^{m *} F_{m k}^{i}\right)
$$

and

$$
P^{* i}{ }_{j k}=:{ }^{*} P_{j k}^{i}+\Delta_{k}{ }^{*} M_{j}^{i}+{ }^{*} M_{j k}^{i},
$$

which give 
Theorem 4.2. Under the given conformal transformation in a space with generalized $(\alpha, \beta)$-metric the entities $R_{j k}^{* i}$ and $P_{j k}^{* i}$ defined by $(4.9 \mathrm{a}, \mathrm{b})$ are conformally invariant.

We know that the $h$-curvature tensor $R_{h j k}^{i}$ is given as [6]:

$$
R_{h j k}^{i}=\mathrm{C}_{(j, k)}\left\{\partial_{k} F_{h j}^{i}-N_{k}^{m}\left(\Delta_{m} F_{h j}^{i}\right)+F_{h j}^{m} F_{m k}^{i}\right\}+C_{h m}^{i} R_{j k}^{m},
$$

implying

$$
\begin{aligned}
{ }^{*} R_{h j k}^{i}=R_{h j k}^{i} & +C_{h m}^{i} I_{j k}^{m}+M_{h m}^{i}{ }^{*} R_{j k}^{m}+\mathrm{C}_{(j, k)}\left\{D_{h j \mid k}^{i}+D_{0 j}^{m}\left(\Delta_{m}{ }^{*} F_{h k}^{i}\right)\right. \\
& \left.+D_{h j}^{m} D_{m k}^{i}\right\}
\end{aligned}
$$

and

$$
{ }^{*} \underline{R}_{h j k}^{i}={ }^{*} R_{h j k}^{i}+{ }^{*} C_{h m}^{i} J_{j k}^{m}-{ }^{*} N_{h j k}^{i},
$$

where

$$
{ }^{*} N_{h j k}^{i}=: \mathrm{C}_{(j, k)}\left\{{ }^{*} U_{h j \| k}^{i}+{ }^{*} b_{k}^{m}\left(\Delta_{m}{ }^{*} \underline{F}_{h j}^{i}\right)+{ }^{*} U_{m k}^{i}{ }^{*} \underline{F}_{h j}^{m}-{ }^{*} U_{h j}^{m *} U_{m k}^{i}\right\} .
$$

From equations (4.5), (4.12) and (4.13) on simplification, we can obtain

$$
\underline{R}_{h j k}^{* i}=R_{h j k}^{* i}+{ }^{*} U_{h j k}^{i},
$$

where

$$
\begin{aligned}
R_{h j k}^{* i}=:{ }^{*} R_{h j k}^{i} & +\mathrm{C}_{(j, k)}\left\{{ }^{*} F_{h j \| k}^{i}+{ }^{*} N_{k}^{m}\left(\Delta_{m}{ }^{*} F_{h j}^{i}\right)\right. \\
& \left.-{ }^{*} C_{h m}^{i}\left({ }^{*} N_{j \| k}^{m}+{ }^{*} N_{j}^{r} \Delta_{r}{ }^{*} N_{k}^{m}\right)\right\}
\end{aligned}
$$

and

$$
{ }^{*} U_{h j k}^{i}=: \complement_{(j, k)}\left\{{ }^{*} C_{h m}^{i}\left({ }^{*} \underline{N}_{k}^{r} \Delta_{r}{ }^{*} b_{j}^{m}+{ }^{*} F_{r j}^{m *} b_{k}^{r}\right)+{ }^{*} N_{k}^{r} \Delta_{r}{ }^{*} U_{h j}^{i}+{ }^{*} U_{r j}^{i}{ }^{*} F_{h k}^{r}\right\},
$$

which leads to

Theorem 4.3. Under the given conformal transformation in a space with generalized $(\alpha, \beta)$-metric the entity $R_{h j k}^{* i}$ is conformally invariant if and only if ${ }^{*} U_{h j k}^{i}$ vanishes.

We know that the $h v$-curvature tensor $P_{h j k}^{i}$ is given by [6]:

$$
P_{h j k}^{i}=\Delta_{k} F_{h j}^{i}-C_{h k \mid j}^{i}+C_{h m}^{i} P_{j k}^{m}
$$

therefore we can obtain

$$
\begin{aligned}
{ }^{*} P_{h j k}^{i}=P_{h j k}^{i} & +M_{h k \| j}^{i}+\Delta_{k} D_{h j}^{i}+{ }^{*} b_{j}^{m} \Delta_{k} C_{h m}^{i}+{ }^{*} C_{h m}^{i} \Delta_{k} D_{0 j}^{m}+M_{h m}^{i} P_{j k}^{m} \\
& +C_{m k}^{i} D_{h j}^{m}-M_{h m}^{i} D_{j k}^{m}-C_{h k}^{m} D_{m j}^{i},
\end{aligned}
$$


where

$$
\begin{aligned}
M_{j k}^{r} & =(2 L \tau)^{-1}\left[\left(h_{j}^{r}-\tau^{-1} l^{r} m_{j}\right) m_{k}+\left(h_{k}^{r}-\tau^{-1} l^{r} m_{k}\right) m_{j}\right. \\
& \left.+h_{j k}\left\{m^{r}-l^{r} \tau^{-1}\left(b^{2}-\beta^{2} / L^{2}\right)\right\}\right] .
\end{aligned}
$$

The curvature tensor ${ }^{*} P_{h j k}^{i}$ on conformal transformation leads to

$$
{ }^{*} \underline{P}_{h j k}^{i}={ }^{*} P_{h j k}^{i}-\Delta_{k}{ }^{*} U_{h j}^{i}+{ }^{*} C_{h m}^{i}\left(\Delta_{k}{ }^{*} b_{j}^{m}+{ }^{*} U_{j k}^{m}\right) .
$$

From equation $(4.20)$, we can easily obtain $\underline{P}_{h j k}^{* i}=P_{h j k}^{* i}$, where

$$
P_{h j k}^{* i}=:{ }^{*} P_{h j k}^{i}+\Delta_{k}{ }^{*} F_{h j}^{i}-{ }^{*} C_{h m}^{i}\left(\Delta_{k}{ }^{*} N_{j}^{m}+{ }^{*} F_{j k}^{m}\right) .
$$

Theorem 4.4. Under the given conformal transformation in a space with generalized $(\alpha, \beta)$-metric the entity $P_{h j k}^{* i}$ defined by $(4.21)$ is conformally invariant.

We know that the $v$-curvature tensor $S_{h j k}^{i}$ is given by [6]

$$
S_{h j k}^{i}=\mathrm{C}_{(j, k)}\left\{\Delta_{k} C_{h j}^{i}+C_{h j}^{m} C_{m k}^{i}\right\}
$$

therefore by virtue of

$$
{ }^{*} C_{j k}^{r}=C_{j k}^{r}+M_{j k}^{r},
$$

and (2.4), the conformal transformation of generalized $v$-curvature tensor, satisfies the invariant property ${ }^{*} \underline{S}_{h j k}^{i}={ }^{*} S_{h j k}^{i}$. Hence we have:

Theorem 4.5. The curvature tensors of a space with generlized $(\alpha, \beta)$-metric under a conformal transformation satisfy equations (4.12) and (4.20) such that entities $\left({ }^{*} C_{h m}^{i} J_{j k}^{m}-{ }^{*} N_{h j k}^{i}\right)$ and $\left\{\Delta_{k}{ }^{*} U_{h j}^{i}-{ }^{*} C_{h m}^{i}\left(\Delta_{k}{ }^{*} b_{j}^{m}+{ }^{*} U_{j k}^{m}\right)\right\}$ are conformally invariant.

Multiplying equation (4.12) by ${ }^{*} y^{j}$ and comparing the resulting equation with (4.4), on simplification, we obtain equation

$$
\mathrm{C}_{(j, k)}\left\{{ }^{*} U_{m j}^{i}{ }^{*} N_{k}^{m}+{ }^{*} b_{j}^{m} \Delta m^{*} b_{k}^{i}\right\}=0 .
$$

which implies:

Theorem 4.6. Under the given conformal transformation in a space with generalized $(\alpha, \beta)$-metric the tensors ${ }^{*} U_{m j}^{i},{ }^{*} N_{k}^{m}$ and ${ }^{*} b_{j}^{m}$ satisfy equation (4.24).

\section{Some special cases}

Case I. Randers' space: The $v$-curvature tensor in a Randers' space is expressed in the following form [5]

$$
{ }^{*} L^{2 *} S_{h i j k}=\mathrm{C}_{(j, k)}\left(h_{h k} m_{i j}+h_{i j} m_{h k}\right),
$$


where the $v$-Ricci tensor is given by

$$
{ }^{*} L^{2 *} S_{i k}=-\left\{(n-1) m^{2} / 4 \tau\right\}{ }^{*} h_{i k}-\{(n-3) / 4\} m_{i} m_{k},
$$

where $m_{i j}=(\tau / 4)\left\{\left(m^{2} / 2\right) h_{i j}+m_{i} m_{j}\right\}$.

From equations (5.1) and (5.2), we can easily obtain

$$
{ }^{*} \underline{S}_{h i j k}=e^{2 \sigma *} S_{h i j k}, \quad{ }^{*} \underline{S}_{i k}={ }^{*} S_{i k} .
$$

Hence we have:

Theorem 5.1. In a Randers' space ${ }^{*} L^{-2 *} S_{h i j k}$ and ${ }^{*} S_{i k}$ are conformally invariant. In a Randers' space the $(v) h v$-torsion tensor is given by $[5]$

$$
{ }^{*} P_{h j k}=h_{h j} P_{k}+h_{j k} P_{h}+h_{k h} P_{j},
$$

where

$$
2 P_{j}=\left({ }^{*} L / L^{2}\right) F_{j 0}+E_{j 0} / L-F_{\beta j}-P l_{j}-G m_{j}
$$

and

$$
G=\left(E_{00}-2 L F_{\beta 0}\right) /\left(2 L^{*} L\right), \quad P=\tau\left(2 G+F_{\beta 0} /^{*} L\right) .
$$

From equation (5.5b), we can easily obtain

$$
\underline{G}=e^{-\sigma}\left[G+\left\{\sigma_{0}\left(\beta-L b^{2}\right)+L \beta \sigma_{a} b^{a}\right\} /\left(2 L^{*} L\right)\right]
$$

and

$$
\underline{P}=e^{-\sigma}\left[P+\beta \sigma_{a} b^{a} /(2 L)+\sigma_{0}\left(2 \beta-L b^{2}\right) /\left(2 L^{2}\right)\right] .
$$

With the help of equations (5.5a) and (5.6a,b), we can obtain on simplification

$$
\begin{aligned}
2 \underline{P}_{j}=2 P_{j} & +\sigma_{0}\left[\tau b_{j}+m_{j}+\left(b^{2}-\beta / L\right)\left(l_{j}+\tau^{-1} m_{j}\right)\right] /(2 L)-\sigma_{j}\left\{\beta(\tau+1)-b^{2} L\right\} / \\
& +(1 / 2) \sigma_{a} b^{a} m_{j}\left(1-\beta /^{*} L\right),
\end{aligned}
$$

which implies for $P_{j} y^{j}=P_{0}$,

$$
\underline{P}_{0}=P_{0}+(1 / 2)\left(b^{2}-\beta / L\right) \sigma_{0} .
$$

From equation (2.11), we can obtain

$$
L\left(\sigma_{a} b^{a}\right)=\left(\partial_{a} \underline{L}\right) \underline{b}^{a}-\left(\partial_{a} L\right) b^{a} .
$$

From equations $(5.6 \mathrm{a}, \mathrm{b})$ with the help of equations $(2.11),(5.7)$ and (5.8) together with $T={ }^{*} L G+2 P_{0} L$, we can obtain

$$
\sigma_{a} b^{a}=2 \beta^{-1}(\underline{T}-T)
$$


From equation (5.7) on simplification we can obtain $\underline{Q}_{j}=Q_{j}$, where

$$
\begin{aligned}
Q_{j}=: 4 P_{j} & -2 L^{-1} P_{0}\left\{\left(\tau b_{j}+m_{j}\right)\left(b^{2}-\beta / L\right)^{-1}-\left(l_{j}+\tau^{-1} m_{j}\right)\right\}+2 T m_{j}(\tau \beta)^{-1} \\
& +L^{-2}\left\{\beta(\tau+1)-b^{2} L\right\}\left(\partial_{j} L\right) .
\end{aligned}
$$

Hence we have:

Theorem 5.2. In a Randers' space entities $L \sigma_{a} b^{a}, \beta \sigma_{a} b^{a}$ and $Q_{j}$ are conformally invariant.

From equations (5.4) and (5.7) with the help of equation (5.11) on simplification we can obtain $\underline{P}^{*}{ }_{h j k}=P^{*}{ }_{h j k}$, where

$$
P^{*}{ }_{h j k}=:{ }^{*} L^{-1}\left[{ }^{*} P_{h j k}+(1 / 4)\left\{h_{h j} Q_{k}+h_{j k} Q_{h}+h_{k h} Q_{j}\right\}\right] .
$$

Hence we have:

Theorem 5.3. In a Randers' space the entity $P^{*}{ }_{h j k}$ defined by (5.12) is conformally invariant.

Cast II. Landsberg space: If Randers' space reduces to a Landsberg space, we can write [5]

$$
{ }^{*} R_{h j k}^{i}=R_{h j k}^{i}+{ }^{*} C_{h r}^{i} R_{0 j k}^{r},
$$

where $R_{h j k}^{i}$ is well known Riemannian curvature tensor.

Taking conformal transformation of (5.13), we can obtain

$$
{ }^{*} \underline{R}_{h j k}^{i}={ }^{*} R_{h j k}^{i}+{ }^{*} C_{h r}^{i} X_{0 j k}^{r}+X_{h j k}^{i},
$$

where

$$
X_{h j k}^{i}=\underline{R}_{h j k}^{i}-R_{h j k}^{i} .
$$

From equation (5.14), we can obtain $\underline{R}_{h j k}^{* i}=R_{h j k}^{* i}$, where

$$
\begin{aligned}
R_{h j k}^{* i}=:{ }^{*} R_{h j k}^{i} & -\mathrm{C}_{(j, k)}\left[\delta_{k}^{i}\left\{R_{h j}-R g_{h j} / 2(n-1)\right\}+g^{i l} g_{h j}\left\{R_{l k}-R g_{l k} / 2(n-1)\right\}\right. \\
& +{ }^{*} C_{h r}^{i}\left[\left\{R_{0 j}-R y_{j} / 2(n-1)\right\}\right. \\
& \left.\left.-y_{j} g^{r l}\left\{R_{l k}-R g_{l k} / 2(n-1)\right\}\right]\right] /(n-2) .
\end{aligned}
$$

Hence we have:

Theorem 5.4. In a Landsberg space the entity $R^{* i}{ }_{h j}$ defined by (5.16) is conformally invariant. 


\section{References}

[1] M. Hashiguchi, On conformal transformations of Finsler metrics, J. Math. Kyoto Univ. 16(1976), 25-50.

[2] H. Izumi, Conformal transformation of Finsler space I, Tensor, N. S. 31(1977), 33-41.

[3] H. Izumi, Conformal transformation of Finsler space II, Tensor, N. S. 34(1980), 337-359.

[4] M. S. Knebelman, Conformal geometry of generalized metric space, Proc. Nat. Acad. Sci. U. S. A., 15(1929), 376-379.

[5] M. Matsumoto, On Finsler spaces with Randers' metric and special forms of important tensors, J. Math. Kyoto Univ. 14(1974), 477-498.

[6] M. Matsumoto, Foundations of Finsler geometry and special Finsler spaces, Kaiseisha Press. Saikawa, Otsu, 520, Japan, 1986.

[7] M. Matsumoto, Theory of Finsler spaces with $(\alpha, \beta)$-metric, Rep. Math. Phys. 31(1992), 43-83.

[8] M. Matsumoto, Conformal changes of Finsler spaces with 1-form metric, Analele Stiitifice Ale Universitatii “A. L. I. Cuza”, Iasi, XL(1994), 1-6.

[9] G. Randers, On an asymmetric metric in the four space of general relativity, Phys. Rev.(2) 59(1941), 195-199.

Department of Mathematics, Shri Ramswaroop Memorial, College of Engineering and Management, Tewarigenj, Faizabad Road, Lucknow-227105, India.

E-mail: sureshrastogi @rediffmail.com

Department of Mathematics, Northern India Engineering College, Faizabad Road, Lucknow227105, India.

E-mail: dwivediashwini@yahoo.co.in 